Published in final edited form as:

Nature. 2014 August 14; 512(7513): 143-144. doi:10.1038/nature13650.

\title{
One cell at a time
}

Edward J. Fox and Lawrence A. Loeb

Department of Pathology, University of Washington, Seattle, Washington 98195-7750, USA.

L.A.L. is also in the Department of Biochemistry, University of Washington

Edward J. Fox: eddiefox@uw.edu; Lawrence A. Loeb: laloeb@uw.edu

\section{Abstract}

Single-cell DNA sequencing of two breast-cancer types has shown extensive mutational variation in individual tumours, confirming that generation of genetic diversity may be inherent in how tumours evolve.

Next-generation DNA sequencing has revolutionized the field of cancer genomics ${ }^{1}$.

Although this sequencing can identify the most frequent mutation in a population of cells, it struggles to resolve the mutational diversity and multiple genomes of the individual cells that comprise a tumour. Achieving DNA sequencing down to the resolution of a single cell has been a long-held dream for understanding the cellular heterogeneity that is inherent in many complex biological systems and, in particular, for delineating the mixture of genomes in human cancers ${ }^{2}$. On page 155 of this issue, Wang et al. ${ }^{3}$ report an innovative sequencing method, termed nuc-seq, that achieves almost complete sequencing of whole genomes in single cells.

As a cell prepares to divide, it replicates the DNA in its nucleus. By sorting and sequencing only the newly 'doubled' nuclei, nuc-seq takes advantage of this duplication to achieve lower rates of sequencing errors than most previous techniques ${ }^{4}$. The authors validated their method using targeted duplex sequencing, a protocol that sequences both strands of DNA to identify mutations at exceptionally high accuracy ${ }^{5}$. They suggest that the use of nuc-seq to sequence single-cell genomes, with validation by targeted deep sequencing, will be instrumental in defining the genomic heterogeneity of cancers.

To demonstrate this, Wang et al. used their technique to sequence the genomes of multiple single cells from two types of human breast cancer, and found that no two individual tumour cells were genetically identical. As well as the large numbers of mutations that are common to the majority of cells in a tumour, the authors uncovered an even greater number of subclonal and de novo mutations (those that are unique to individual cells). They also present estimates, derived from mathematical models, of mutation rates of single cells within tumours. On the basis of these models, they show that distinct types of DNA alteration seem to accumulate at different rates in different tumours, and suggest that two separate 'mutational clocks' operate in cancer. Large-scale, structural changes in DNA (such as amplification and deletion of large blocks of DNA) probably occur early in tumour development, in punctuated bursts of evolution, whereas point mutations may accumulate more gradually, generating extensive subclonal diversity. The authors' findings indicate that slower-growing 'luminal' breast-cancer cells exhibit relatively low mutation rates, whereas 
cells from clinically more aggressive, 'triple-negative' breast cancers have mutation rates that are 13 times greater than in normal cells.

Nuc-seq and comparable single-cell sequencing methods ${ }^{6-9}$ will allow a more detailed understanding of mutational heterogeneity in individual tumours, and will influence our understanding of how cancers evolve and our approach to their treatment. In particular, mutational diversity within a tumour is likely to be predictive of whether resistance to a particular chemotherapy will emerge during treatment, because mutations in genes that render cells resistant to specific drugs may exist before initiation of therapy. This has previously been documented for the failure of certain molecularly tailored cancer treatments ${ }^{10}$. Such findings also reinforce the fact that single, bulk sampling of a tumour a strategy that is commonly used to select targeted therapies — is not representative of the tumour as a whole.

The total number of mutations that a tumour genome carries, including those present in only a small subset of cells, may in fact underlie the aggressiveness of different cancer subtypes. For example, the extent of genetic diversity within a tumour, and its divergence from normal tissue, probably influences the ability of the immune system to distinguish malignant cells from normal cells. Identifying the mechanisms by which cancer cells generate mutational heterogeneity may therefore present previously unexplored therapeutic targets.

An array of techniques to analyse individual cells has now been developed. It remains to be seen, however, just how robust nuc-seq and other single-cell genomics techniques, such as MALBAC $^{6}$, will prove to be. For example, many cancer cells are aneuploid (they carry abnormal numbers of chromosomes), and the application of nuc-seq may be restricted to cancers that do not exhibit aneuploidy. Also, although the cost of genome sequencing continues to decline (albeit more slowly now than in the past), the cost of single-cell genomics and the complexities of the bioinformatic analyses involved are still formidable.

In our quest to decipher cancer genomes, the advent of single-cell sequencing marks a technical milestone. It crystallizes the concept that the genome of each tumour is dynamic and highly diverse, whether we are comparing cancer genomes between tumours of different patients, between anatomically distinct regions of a tumour within a patient or even between individual cells within the same tumour (Fig. 1). Single-cell sequencing will allow us to detect rare mutant subpopulations hidden within cancers that could expand and lead to drug resistance, and thus to avoid unnecessary and potentially harmful administration of ineffective, toxic therapies. Ultimately, the exceptional plasticity of the tumour genome may well prove to be a key characteristic of cancer $^{11}$ and a major, as yet untapped, therapeutic vulnerability.

\section{References}

1. Stratton MR, Campbell PJ, Futreal PA. Nature. 2009; 458:719-724. [PubMed: 19360079]

2. Nature Meth. 2014; 11:1.

3. Wang Y, et al. Nature. 2014; 512:155-160. [PubMed: 25079324]

4. Navin N, et al. Nature. 2011; 472:90-94. [PubMed: 21399628]

5. Schmitt MW, et al. Proc. Natl Acad. Sci. USA. 2012; 109:14508-14513. [PubMed: 22853953] 
6. Zong C, Lu S, Chapman AR, Xie XS. Science. 2012; 338:1622-1626. [PubMed: 23258894]

7. Shapiro E, Biezuner T, Linnarsson S. Nature Rev. Genet. 2013; 14:618-630. [PubMed: 23897237]

8. Xu X, et al. Cell. 2012; 148:886-895. [PubMed: 22385958]

9. Hou Y, et al. Cell. 2012; 148:873-885. [PubMed: 22385957]

10. Tougeron D, et al. Ann. Oncol. 2013; 24:1267-1273. [PubMed: 23293113]

11. Loeb LA, Springgate CF, Battula N. Cancer Res. 1974; 34:2311-2321. [PubMed: 4136142] 


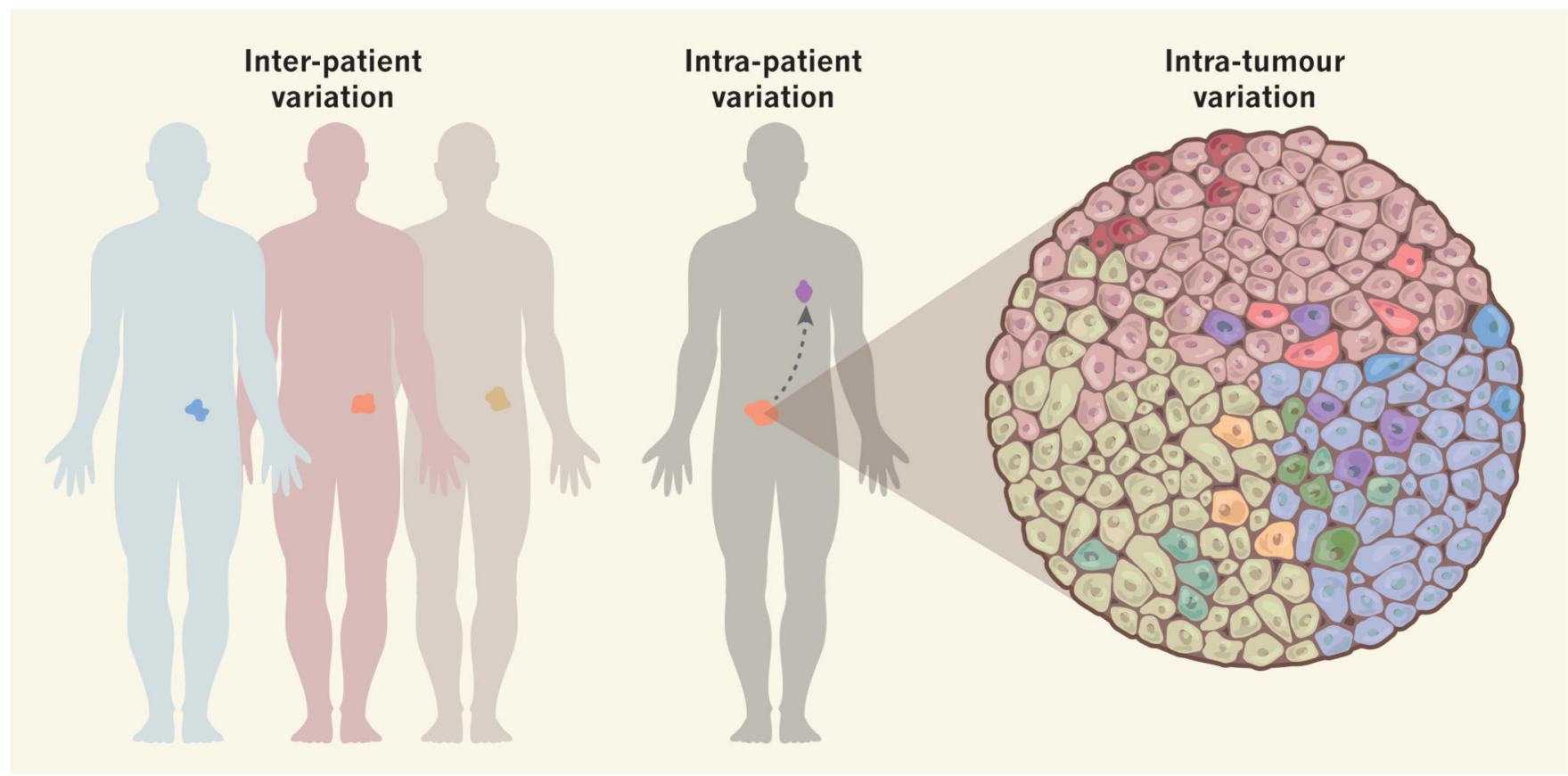

Figure 1.

The genetic characteristics of cancers vary between patients, between primary and metastatic tumours in a single patient, and between the individual cells of a tumour. Wang et $a l{ }^{3}$ present a single-cell, whole-genome sequencing technique that will allow a better understanding of genetic heterogeneity within individual tumours. 